PROCEEDINGS OF THE

AMERICAN MATHEMATICAL SOCIETY

Volume 134, Number 9, September 2006, Pages 2755-2765

S 0002-9939(06)08607-2

Article electronically published on April 13, 2006

\title{
A PREVALENT TRANSVERSALITY THEOREM FOR LIPSCHITZ FUNCTIONS
}

\author{
CHRIS SHANNON
}

(Communicated by Jonathan M. Borwein)

\begin{abstract}
This paper provides a version of the transversality theorem for a class of Lipschitz functions of the form $f: \mathbf{R}^{n} \times C \rightarrow \mathbf{R}^{n}$, where $C$ is a convex subset of a normed vector space $Z$ indexing the parameters in the problem. The set $C$ may be infinite-dimensional, and the notion of generic used is the measure-theoretic notion of prevalence introduced by Hunt, Sauer and Yorke (1992) and Christensen (1974). This paper also provides some results on sensitivity analysis for solutions to locally Lipschitz equations.
\end{abstract}

\section{INTRODUCTION}

This paper studies a class of problems of the following form: for a given Lipschitz function $f: \mathbf{R}^{n} \times Z \rightarrow \mathbf{R}^{n}$ and a fixed value $y \in \mathbf{R}^{n}$, how big is the subset of parameters $z$ in a set $C \subset Z$ for which all solutions $x$ to the equation

$$
f(x, z)=y
$$

are locally unique and vary in a Lipschitz manner with $z$ ? That is, how big is the set of parameters in $C$ for which the solutions to this equation are "determinate"?

To address this question, this paper first provides some results on sensitivity analysis for solutions to locally Lipschitz equations. These ideas are then used to establish a version of the transversality theorem for a class of Lipschitz functions of the form $f: \mathbf{R}^{n} \times C \rightarrow \mathbf{R}^{n}$, where $C$ is a convex subset of a normed vector space $Z$. The set $C$ may be infinite-dimensional, and the notion of generic used is the measure-theoretic notion of prevalence introduced by Christensen [3] and developed by Hunt, Sauer and Yorke 9], and the extension of this concept to relative prevalence developed by Anderson and Zame [1].

\section{Regular values of Lipschitz Functions}

We start with some preliminary results regarding sensitivity analysis for solutions to locally Lipschitz equations. Several different notions of regular point and regular value of a Lipschitz function will be used in this paper, each giving rise to different

Received by the editors April 25, 2003.

2000 Mathematics Subject Classification. Primary 58C05, 49J52, 90C31; Secondary 58E17.

Key words and phrases. Prevalence, transversality, Lipschitz functions, nonsmooth analysis.

Thanks to Bob Anderson, Don Brown, Max Stinchcombe and Bill Zame for helpful comments and conversations concerning this paper. The financial support of the National Science Foundation under grant SBR 98-18759, the Miller Institute, and an Alfred P. Sloan Foundation Research Fellowship is gratefully acknowledged. 
properties of the set of solutions. The basic definition which will be the focus for most of the results of this paper is the following.

Definition 2.1. Given a function $g: \mathbf{R}^{m} \rightarrow \mathbf{R}^{n}$, a point $x \in \mathbf{R}^{m}$ is a regular point of $g$ if $g$ is differentiable at $x$ and $D g(x)$ is surjective. A value $y \in \mathbf{R}^{n}$ is a regular value of $g$ if every point $x \in g^{-1}(y)$ is a regular point of $g$.

The main application of this concept here will be to the case in which $g=f(\cdot, z)$ for a locally Lipschitz function $f: \mathbf{R}^{n} \times Z \rightarrow \mathbf{R}^{n}$, where $Z$ is a Banach space and $z \in Z$. In this case, if $y \in \mathbf{R}^{n}$ is a regular value of $f(\cdot, \bar{z})$ for some fixed $\bar{z} \in Z$, then every solution $x \in \mathbf{R}^{n}$ to the equation $f(x, \bar{z})=y$ is locally unique. Unlike the case in which $f$ is $C^{1}$, if $f$ is only locally Lipschitz, then this condition is not sufficient to imply that the solution set will remain locally single-valued as $z$ changes. A simple counterexample is provided by the function $g: \mathbf{R} \rightarrow \mathbf{R}$ given by $g(x)=x^{2} \sin (1 / x)+x$, which is Lipschitz continuous and for which 0 is a regular point, but which is not one-to-one on any neighborhood of 0 . Sensitivity analysis for the set of solutions will then rely on derivatives for multivalued maps and on some of the techniques developed in the recent work on nonsmooth or variational analysis (see e.g. Rockafellar [12, 13, Rockafellar and Wets 14, Clarke, Ledyaev, Stern and Wolenski [5]).

The derivatives for multivalued maps that are most useful in this problem are based on Painlevé-Kuratowski convergence of sets. If $\left\{A_{t}: t \in T\right\}$ is a net of sets in a topological space, then

$$
\limsup A_{t} \equiv\left\{a: a=\lim _{n} a_{t_{n}}, a_{t_{n}} \in A_{t_{n}}, n=1,2, \ldots\right\}
$$

and

$$
\liminf A_{t} \equiv\left\{a: a=\lim _{t} a_{t}, a_{t} \in A_{t}, t \in T\right\} .
$$

Note that for a given net $\left\{A_{t}\right\}$ one or both of these sets may be empty. If $\limsup A_{t}=\liminf A_{t}$, then $\left\{A_{t}\right\}$ has a limit and $\lim _{t} A_{t} \equiv \limsup A_{t}=\lim \inf A_{t}$.

The main derivative used here is the contingent derivative, introduced by Aubin 2]. Given a multivalued map $H: X \rightrightarrows Y$ where $X$ and $Y$ are normed spaces, the contingent derivative of $H$ at a point $(x, y) \in \operatorname{graph}(H)$ is denoted $D H(x \mid y)$ and is the mapping whose graph is the contingent cone to the graph of $H$ at $(x, y)$, that is,

$$
\text { graph } D H(x \mid y)=\limsup _{t \downarrow 0} \frac{1}{t}[\operatorname{graph} H-(x, y)] .
$$

Rockafellar [12] provides an important strengthening of this condition in the spirit of Hadamard differentiability by requiring that the limit exist in the above expression and that this limit not depend on the way a particular direction is approached. More precisely, $H$ is semi-differentiable at $(x, y)$ if

$$
\lim _{\substack{t \downarrow 0 \\ w^{\prime} \rightarrow w}} \frac{1}{t}\left[H\left(x+t w^{\prime}\right)-y\right]
$$

exists for all $w \in X$. When this limit exists it is simply equal to the contingent derivative at $w, D H(x \mid y)(w)$.

King and Rockafellar [10] show that the single-valuedness of the contingent derivative provides a regularity condition strong enough to imply that the set of solutions 
to generalized equations is semi-differentiable and locally Lipschitz in the following sense. A multivalued map $H: X \rightrightarrows Y$ between two normed spaces is upper Lipschitzian at $\bar{x} \in X$ if there exists $\lambda>0$ and a neighborhood $\Omega$ of $\bar{x}$ such that

$$
H(x) \subset H(\bar{x})+\lambda\|x-\bar{x}\| B \quad \forall x \in \Omega,
$$

where $B$ is the unit ball in $Y$. A multivalued map that is upper Lipschitzian at a point $\bar{x}$ exhibits a strong type of stability in a neighborhood of that point: for every $x \in \Omega$, if $y \in H(x)$, then there exists $\bar{y} \in H(\bar{x})$ such that $\|y-\bar{y}\| \leq \lambda\|x-\bar{x}\|$.

To study the behavior of the solutions of the equation $f(x, z)=y$ as $z$ varies, let the solution map $E: Z \rightrightarrows \mathbf{R}^{n}$ be defined by

$$
E(z) \equiv\left\{x \in \mathbf{R}^{n}: f(x, z)=y\right\} .
$$

For these results, we suppose that the parameterization itself is sufficiently smooth, in the following sense.

Definition 2.2. Let $X, Y$, and $Z$ be topological vector spaces. A function $f$ : $X \times Z \rightarrow Y$ is Fréchet equi-differentiable in $z$ if for every $x \in X, f(x, \cdot)$ is differentiable at every point $z \in Z$, and $D_{z} f(B)$ is bounded for every bounded subset $B \subset X \times Z$.

For example, if $X$ and $Z$ are locally compact, $f(x, \cdot)$ is differentiable in $z$ for each $x \in X$ and $D_{z} f$ is continuous on $X \times Z$, then 1

The first result below shows that if $y$ is a regular value of $f(\cdot, \bar{z})$, then the solution map $E(\cdot)$ is locally upper Lipschitzian at $\bar{z}$ and semi-differentiable provided the parameterization is equi-differentiable.

Theorem 2.3. Let $Z$ be a normed vector space. Let $f: \mathbf{R}^{n} \times Z \rightarrow \mathbf{R}^{n}$ be locally Lipschitz and Fréchet equi-differentiable in $z$. Let $y \in \mathbf{R}^{n}$ be a regular value of $f(\cdot, \bar{z})$ for some $\bar{z} \in Z$. For each $\bar{x} \in E(\bar{z})$ there exists a neighborhood $U$ such that $U \cap E(\bar{z})=\{\bar{x}\}$ and such that $U \cap E(\cdot)$ is upper Lipschitzian at $\bar{z}$. Moreover, $E$ is semi-differentiable at $\bar{z}$ relative to $\bar{x}$ and for each $z \in Z$,

$$
D E(\bar{z} \mid \bar{x})(z)=\lim \sup _{t \downarrow 0} \frac{1}{t}[E(\bar{z}-t z)-\bar{x}] .
$$

Every selection $x(\cdot) \in E(\cdot) \cap U$ is upper Lipschitzian and semi-differentiable at $\bar{z}$ with $D x(\bar{z} \mid \bar{x})=D E(\bar{z} \mid \bar{x})$.

Proof. First, $D E(\bar{z} \mid \bar{x})(0) \subset\{0\}$. To see this, note that by definition $(0, v) \in$ graph $D E(\bar{z} \mid \bar{x}) \Longleftrightarrow \exists\left(w_{t}, v_{t}\right)$ such that $\left(w_{t}, v_{t}\right)=\frac{1}{t}\left(\left(z_{t}, x_{t}\right)-(\bar{z}, \bar{x})\right)$, where $\left(z_{t}, x_{t}\right) \in$ graph $E$, i.e., where $f\left(x_{t}, z_{t}\right)=y$ for all $t$, and such that $\left(w_{t}, v_{t}\right) \rightarrow(0, v)$. Note that $z_{t} \rightarrow \bar{z}$ and $x_{t} \rightarrow \bar{x}$ as $t \rightarrow 0$. Now let $\Delta z_{t}=z_{t}-\bar{z}$ and $\Delta x_{t}=x_{t}-\bar{x}$. Since $y$ is a regular value of $f(\cdot, \bar{z})$ and $f$ is Fréchet equi-differentiable in $z, f$ is (Fréchet) differentiable at $(\bar{x}, \bar{z})$. Then

$$
f\left(\bar{x}+\Delta x_{t}, \bar{z}+\Delta z_{t}\right)-f(\bar{x}, \bar{z})-D f(\bar{x}, \bar{z})\left(\Delta x_{t}, \Delta z_{t}\right)=o\left(\left\|\left(\Delta x_{t}, \Delta z_{t}\right)\right\|\right),
$$

which implies that

$$
\begin{aligned}
D f(\bar{x}, \bar{z})\left(\Delta x_{t}, \Delta z_{t}\right) & =o\left(\left\|\left(\Delta x_{t}, \Delta z_{t}\right)\right\|\right)+f\left(\bar{x}+\Delta x_{t}, \bar{z}+\Delta z_{t}\right)-f(\bar{x}, \bar{z}) \\
& =o\left(\left\|\left(\Delta x_{t}, \Delta z_{t}\right)\right\|\right)+0
\end{aligned}
$$

\footnotetext{
${ }^{1}$ An alternative sufficient condition for these results is that $f(x, \cdot)$ is continuously differentiable for each $x \in X$.
} 
since $f\left(\bar{x}+\Delta x_{t}, \bar{z}+\Delta z_{t}\right)=f\left(x_{t}, z_{t}\right)=y=f(\bar{x}, \bar{z})$ for every $t$. So

$$
\begin{aligned}
\operatorname{Df}(\bar{x}, \bar{z}) \frac{\left(\Delta x_{t}, \Delta z_{t}\right)}{\left\|\left(\Delta x_{t}, \Delta z_{t}\right)\right\|} & =\frac{o\left(\left\|\left(\Delta x_{t}, \Delta z_{t}\right)\right\|\right)}{\left\|\left(\Delta x_{t}, \Delta z_{t}\right)\right\|} \\
\Rightarrow D f(\bar{x}, \bar{z}) \frac{\left(\frac{\Delta x_{t}}{t}, \frac{\Delta z_{t}}{t}\right)}{\left\|\left(\frac{\Delta x_{t}}{t}, \frac{\Delta z_{t}}{t}\right)\right\|} & =\frac{o\left(\left\|\left(\Delta x_{t}, \Delta z_{t}\right)\right\|\right)}{\left\|\left(\Delta x_{t}, \Delta z_{t}\right)\right\|} \\
\Rightarrow D f(\bar{x}, \bar{z})\left(\frac{\Delta x_{t}}{t}, \frac{\Delta z_{t}}{t}\right) & =\left\|\left(\frac{\Delta x_{t}}{t}, \frac{\Delta z_{t}}{t}\right)\right\| \frac{o\left(\left\|\left(\Delta x_{t}, \Delta z_{t}\right)\right\|\right)}{\left\|\left(\Delta x_{t}, \Delta z_{t}\right)\right\|} .
\end{aligned}
$$

As $t \rightarrow 0,\left(\frac{\Delta x_{t}}{t}, \frac{\Delta z_{t}}{t}\right)=\left(v_{t}, w_{t}\right) \rightarrow(v, 0)$ and the right-hand side above goes to 0 , so this last equation implies that $D f(\bar{x}, \bar{z})(v, 0)=0$. Thus $D_{x} f(\bar{x}, \bar{z}) v=0$. Because $y$ is a regular value of $f(\cdot, \bar{z}), D_{x} f(\bar{x}, \bar{z})$ is nonsingular, which implies that $v=0$. Thus $D E(\bar{z} \mid \bar{x})(0) \subset\{0\}$ as desired.

Now we show that for $w \in Z$ arbitrary, $D E(\bar{z} \mid \bar{x})(w)$ is at most single-valued. Suppose $v \in D E(\bar{z} \mid \bar{x})(w)$. Then there exists $\left(q_{t}, v_{t}\right)$ such that

$$
\left(q_{t}, v_{t}\right)=\frac{1}{t}\left(\left(z_{t}, x_{t}\right)-(\bar{z}, \bar{x})\right),
$$

where $\left(z_{t}, x_{t}\right) \in$ graph $E$, i.e., where $f\left(x_{t}, z_{t}\right)=y$ for all $t$, and such that $\left(q_{t}, v_{t}\right) \rightarrow$ $(w, v)$. As above note that $z_{t} \rightarrow \bar{z}$ and $x_{t} \rightarrow \bar{x}$. Repeating the argument above shows that $D f(\bar{x}, \bar{z})\left(\frac{\Delta x_{t}}{t}, \frac{\Delta z_{t}}{t}\right)=D f(\bar{x}, \bar{z})\left(v_{t}, q_{t}\right) \rightarrow 0$ as $t \rightarrow 0$. Thus $D f(\bar{x}, \bar{z})(v, w)=0$. Now if there exist $v_{1}, v_{2}$ such that $v_{1}, v_{2} \in \operatorname{graph} D E(\bar{z} \mid \bar{x})(w), \quad D f(\bar{x}, \bar{z})$ $\left(\left(v_{1}, w\right)-\left(v_{2}, w\right)\right)=0$, i.e., $D f(\bar{x}, \bar{z})\left(v_{1}-v_{2}, 0\right)=0$. By the same argument as above, this implies that $v_{1}-v_{2}=0$, i.e., $v_{1}=v_{2}$.

Finally, we must show that if $U^{\prime}$ is a neighborhood of $\bar{x}$, then $E^{-1}\left(U^{\prime}\right)$ is a neighborhood of $\bar{z}$. Since $D E(\bar{z} \mid \bar{x})(0) \subset\{0\}$, there exists a neighborhood $U$ of $\bar{x}$ such that $E(\bar{z}) \cap U=\{\bar{x}\}$ by King and Rockafellar [10, Proposition 2.1]. Since $y$ is a regular value of $f(\cdot, \bar{z}),|d(f(\cdot, \bar{z}), U, y)|=1$ where $d(\cdot)$ denotes Brouwer's degree, by Shannon [15, Theorem 9]. By the domain decomposition property of Brouwer's degree and the fact that $E(\bar{z}) \cap U=\{\bar{x}\},\left|d\left(f(\cdot, \bar{z}), U \cap U^{\prime}, y\right)\right|=1$. Hence $\bar{x}$ is an essential solution of the equation $f(x, \bar{z})=y$ on $U \cap U^{\prime}$ (see, e.g., Fort [8]). This yields a neighborhood $W$ of graph $f(\cdot, \bar{z})$ such that if $g: U \cap U^{\prime} \rightarrow \mathbf{R}^{n}$ is any continuous function with graph $g \subset W$, then $g^{-1}(y) \cap\left(U \cap U^{\prime}\right) \neq \emptyset$. Since $f$ is Lipschitz in $z$, we can choose $r>0$ such that for $z \in B_{r}(\bar{z})$, graph $\left.f(\cdot, z)\right|_{U \cap U^{\prime}} \subset W$. Thus for all $z \in B_{r}(\bar{z}), \exists x \in U \cap U^{\prime}$ such that $f(x, z)=y$, which implies that $B_{r}(\bar{z}) \subset E^{-1}\left(U \cap U^{\prime}\right) \subset E^{-1}\left(U^{\prime}\right)$. Now the result follows from King and Rockafellar [10, Proposition 2.2].

If the function $f$ is $C^{1}$ instead of merely locally Lipschitz continuous, then much stronger results can be derived when $y$ is a regular value of $f$, in particular versions of the transversality theorem. To develop analogues of these results in the case when $f$ is only Lipschitz continuous requires different notions of regular value which build on the generalized Jacobian or Clarke derivative of a Lipschitz function and utilize Clarke's implicit function theorem for Lipschitz functions (Clarke [4]).

If $f: \mathbf{R}^{m} \rightarrow \mathbf{R}^{n}$ is locally Lipschitz continuous, then its generalized Jacobian at a point $x \in \mathbf{R}^{m}$, denoted $\partial f(x)$, is given by

$$
\partial f(x)=\overline{c o}\left\{A: A=\lim _{\substack{x^{\prime} \rightarrow x \\ x^{\prime} \in D_{f}}} D f\left(x^{\prime}\right)\right\},
$$


where $\overline{c o}(B)$ denotes the closed convex hull of the set $B$, and $D_{f}$ denotes the set of points at which $f$ is differentiable. In particular, note that the generalized Jacobian may be multi-valued at some points. The generalized Jacobian is said to be of maximal rank at $x$ if every element of $\partial f(x)$ is of maximal rank. For our results, we will need a strengthening of this condition.

Definition 2.4. Let $f: \mathbf{R}^{m} \rightarrow \mathbf{R}^{n}$ be locally Lipschitz continuous, where $m \geq n$. The generalized Jacobian $\partial f$ is uniformly of maximal rank at $x$ if there exists $\left(v_{1}, \ldots, v_{n}\right)$ such that $\partial_{\left(v_{1}, \ldots, v_{n}\right)} f(x)$ has maximal rank.

For the generalized gradient to be uniformly of maximal rank at a point $x$ then means not only that every element of $\partial f(x)$ is of maximal rank, but that the generalized partial gradient of $f$ with respect to some fixed set of variables $v$ is of maximal rank. This notion of nonsingularity suggests the following stronger notion of regular value.

Definition 2.5. Let $f: \mathbf{R}^{m} \rightarrow \mathbf{R}^{n}$ be locally Lipschitz continuous. A vector $y \in \mathbf{R}^{n}$ is a uniformly regular value of $f$ if for each $x \in f^{-1}(y)$ the generalized Jacobian $\partial f(x)$ is uniformly of maximal rank at $x$.

For uniformly regular values a Lipschitz version of the preimage theorem holds; that is, the preimage of a uniformly regular value is a Lipschitz manifold 2

Theorem 2.6. Let $f: \mathbf{R}^{n} \times \mathbf{R}^{k} \rightarrow \mathbf{R}^{n}$ be locally Lipschitz. If $y$ is a uniformly regular value of $f$, then $f^{-1}(y)$ is a $k$-dimensional Lipschitz manifold or is empty.

Proof. Assume $f^{-1}(y) \neq \emptyset$, and let $(x, z) \in f^{-1}(y)$. Without loss of generality, suppose $\partial_{x} f(x, z)$ has maximal rank. By Clarke's implicit function theorem (Clarke [4), there exist neighborhoods $V$ of $z$ and $W$ of $x$ and a Lipschitz function $g$ : $V \rightarrow W$ such that for all $z^{\prime} \in V, f\left(g\left(z^{\prime}\right), z^{\prime}\right)=y$ and such that $x^{\prime} \in W$ and $f\left(x^{\prime}, z^{\prime}\right)=y$ iff $x^{\prime}=g\left(z^{\prime}\right)$. Then graph $(g)=\{(g(z), z): z \in V\} \subset f^{-1}(y)$ and $f^{-1}(y) \cap(W \times V) \subset$ graph $(g)$. Since graph $(g) \subset W \times V$, this implies $f^{-1}(y) \cap(W \times V)=$ graph $(g)$. Moreover, graph $(g)$ is a $k$-dimensional Lipschitz manifold, which is easily seen by considering the transition functions $\phi: \mathbf{R}^{n} \times V \rightarrow$ $\mathbf{R}^{n} \times V$ defined by $\phi(x, z)=(x-g(z), z)$. Thus $f^{-1}(y)$ is a $k$-dimensional Lipschitz manifold.

The transversality theorems that are developed in the next section rely on an additional, slightly stronger notion of regular value, which requires that the partial derivative with respect to a particular set of parameters is nonsingular at every solution. To formalize this, let $f: \mathbf{R}^{n} \times Z \rightarrow \mathbf{R}^{n}$, where $Z$ is a Banach space, and let $V$ be an $n$-dimensional subspace of $Z$. Since $V$ is finite-dimensional, $Z=V \oplus V^{\perp}$. For each fixed $z \in Z$, define $f^{z}: \mathbf{R}^{n} \times V \rightarrow \mathbf{R}^{n}$ by $f^{z}(x, w)=f\left(x, w+z_{V^{\perp}}\right)$ for each $(x, w) \in \mathbf{R}^{n} \times V$, where $z=z_{V}+z_{V^{\perp}}$ for $z_{V} \in V$ and $z_{V^{\perp}} \in V^{\perp}$. Note that $f^{z}(x, w)=y$ for some $z \in Z$ and $(x, w) \in \mathbf{R}^{n} \times V$ if and only if $w=z_{V}$ and $(x, z) \in f^{-1}(y)$. Let $\lambda_{V}$ denote Lebesgue measure on $V$

\footnotetext{
${ }^{2}$ A set $\mathcal{M}$ is a Lipschitz manifold if it is a Hausdorff topological space such that for every $x \in \mathcal{M}$, there exists an open neighborhood $W_{x}$ of $x$ and a homeomorphism $\phi_{x}: W_{x} \rightarrow \mathbf{R}^{n}$ such that both $\phi_{x}$ and $\phi_{x}^{-1}$ are Lipschitz continuous.

${ }^{3}$ More precisely, let $T: V \rightarrow \mathbf{R}^{n}$ be a linear isomorphism and set $\lambda_{V}(A)=\lambda_{n}(T(A))$ for each $A \subset V$, where $\lambda_{n}$ is standard Lebesgue measure on $\mathbf{R}^{n}$. While $T$ is not unique and the measure $\lambda_{V}$ does depend on the choice of isomorphism, all such measures are mutually absolutely continuous. Since all such measures have the same sets of measure 0 , the choice of isomorphism is irrelevant here.
} 
Definition 2.7. Let $f: \mathbf{R}^{n} \times Z \rightarrow \mathbf{R}^{n}$ be locally Lipschitz continuous, where $Z$ is a Banach space. Let $C \subset Z$ be a convex Borel set. A vector $y \in \mathbf{R}^{n}$ is a strongly regular value of $f$ relative to $C$ if there exists an $n$-dimensional subspace $V$ of $Z$ such that $\partial_{w} f^{z}\left(x, z_{V}\right)$ has maximal rank for each $(x, z) \in f^{-1}(y)$ and such that $\lambda_{V}(C+a)>0$ for some $a \in Z$. If $C=Z, y$ is called a strongly regular value of $f$.

As both are based on the Clarke derivative, strong regularity and uniform regularity are nested: for a fixed function $f: \mathbf{R}^{n} \times Z \rightarrow \mathbf{R}^{n}$, every strongly regular value is uniformly regular. Regularity, strong regularity and uniform regularity are not necessarily nested, however, as regularity is based on properties of the classical derivative, which does not necessarily coincide with the Clarke derivative at an arbitrary point of differentiability. The next section develops a parametric version of the transversality theorem that links these concepts in answering the question posed initially: strong regularity will lead to generic regularity, and hence generic local uniqueness and Lipschitz stability of the solution set.

\section{A prevalent transversality theorem}

When the parameter value $\bar{z}$ is chosen so that $y$ is a regular value of the locally Lipschitz function $f(\cdot, \bar{z})$, the solution set displays strong stability and directional derivative properties in a neighborhood of $\bar{z}$, as the results of the previous section demonstrate. How large is the set of parameter values for which these conclusions hold? Is this determinacy "typical" or "rare"? Answering this question requires a version of the transversality theorem which is applicable to Lipschitz functions. This section develops such results for both finite-dimensional and infinite-dimensional settings.

A difficulty in establishing the genericity of regular values in parametric settings stems from the fact that the preimage of a regular value must consist only of points at which the function $f$ is differentiable. For any given value of $z$, the set

$$
D_{z} \equiv\left\{x \in \mathbf{R}^{n}: f(\cdot, z) \text { is not differentiable at } x\right\}
$$

has Lebesgue measure zero by Rademacher's theorem. Some control over the dependence of this set on the parameter $z$ is needed, however, to rule out cases in which there exists a large subset of $Z$ on which $f^{-1}(y) \cap\left(D_{z} \times\{z\}\right) \neq \emptyset$.

To handle this difficulty, introduce the following notion.

Definition 3.1. A function $f: \mathbf{R}^{n} \times Z \rightarrow \mathbf{R}^{n}$ is tame if $\exists D \subset \mathbf{R}^{n}$ such that $\forall z \in Z, D=\left\{x \in \mathbf{R}^{n}: f(\cdot, z)\right.$ is not differentiable at $\left.x\right\}$.

A simple example of a class of such functions takes the form $f_{i} \equiv h_{i}^{1}+h_{i}^{2} g_{i}$ for $i=1, \ldots, n$, for functions $h^{1}: \mathbf{R}^{n} \rightarrow \mathbf{R}^{n}, h_{i}^{2}: \mathbf{R}^{n} \rightarrow \mathbf{R}$ and $g_{i}: Z \rightarrow \mathbf{R}$ for each $i=1, \ldots n$, where $g$ is continuously Fréchet differentiable. See also Remark 3.3 and Section 4 .

The first result below considers the case in which the parameter space $Z$ is $n$-dimensional. This finite-dimensional result will provide the basis for the final infinite-dimensional theorem.

Lemma 3.2. Let $f: \mathbf{R}^{n} \times \mathbf{R}^{n} \rightarrow \mathbf{R}^{n}$ be locally Lipschitz, equi-differentiable in $z$, and tame. If $y$ is a strongly regular value of $f$, then

$$
S \equiv\left\{z \in \mathbf{R}^{n}: y \text { is not a regular value of } f(\cdot, z)\right\}
$$

has Lebesgue measure 0. 
Proof. By Theorem 2.6, $f^{-1}(y)$ is an $n$-dimensional Lipschitz manifold. Let $M=$ $f^{-1}(y)$ and let $\pi: M \rightarrow \mathbf{R}^{n}$ be projection onto the last $n$ factors, so $\pi(x, z)=z$. Given $(\bar{x}, \bar{z}) \in M$, choose a neighborhood $W$ on which $M \cap W=$ graph $g$, where $g: \mathbf{R}^{n} \rightarrow \mathbf{R}^{n}$ is Lipschitz. So $M \cap W=\{(x, g(x)): x \in A\}$ for some open set $A \subset \mathbf{R}^{n}$ with $\bar{x} \in A$. Since there is a countable cover of $M$ by such neighborhoods, it suffices to restrict attention to this neighborhood.

Let $\phi(x)=(x, g(x))$. On $A$ consider $\pi \circ \phi: A \rightarrow \mathbf{R}^{n}$; note that $\pi \circ \phi=g$. Let $P_{1} \equiv\left\{z \in \mathbf{R}^{n}: z\right.$ is a regular value of $\left.g\right\}$. Since $g: \mathbf{R}^{n} \rightarrow \mathbf{R}^{n}$ is Lipschitz, $P_{1}$ has full measure in $\mathbf{R}^{n}$ (e.g., Rader [11, Lemma 2]). Let

$$
P_{2} \equiv\left\{z \in \mathbf{R}^{n}: \phi \text { is differentiable at } \phi^{-1}(x, z) \forall(x, z) \in M \cap W\right\} .
$$

Then $P_{2}^{c} \subset g(B)$, where $B \equiv\left\{x \in \mathbf{R}^{n}: \phi\right.$ is not differentiable at $\left.x\right\}$. By definition $B=\left\{x \in \mathbf{R}^{n}: g\right.$ is not differentiable at $\left.x\right\}$, so $P_{1} \subset P_{2}$. Now let $P_{3}=g(D)^{c}$, where $D \equiv\left\{x \in \mathbf{R}^{n}: D_{x} f(x, z)\right.$ does not exist for some $\left.z\right\}$. By tameness,

$$
D=\left\{x \in \mathbf{R}^{n}: f(\cdot, \hat{z}) \text { is not differentiable at } x\right\}
$$

for a fixed $\hat{z} \in X$. For a fixed $\hat{z}, f(\cdot, \hat{z}): \mathbf{R}^{n} \rightarrow \mathbf{R}^{n}$ is a locally Lipschitz function, so $D$ has measure 0 , and then so does $g(D)$, again because $g$ is a Lipschitz function from $\mathbf{R}^{n}$ to $\mathbf{R}^{n}$. Thus $P_{3}^{c}=g(D)$ has measure 0 .

Now define $P=P_{1} \cap P_{3}$; by the above arguments $P$ has full measure. Let $z \in P$. It suffices to show that $y$ is a regular value of $f(\cdot, z)$. Choose $\bar{x}$ such that $(\bar{x}, z) \in M \cap W$. Let $r \in \mathbf{R}^{n}$ be arbitrary. Since $z \in P_{3}$ and $f$ is equi-differentiable in $z, f$ is differentiable at $(\bar{x}, z)$. Let $D f(\bar{x}, z)=\left(D_{x}, D_{z}\right)$. We must show that $D_{x}$ has full rank. Since $y$ is a uniformly regular value of $f$ and $D f(\bar{x}, z) \in \partial f(\bar{x}, z)$, there exists $\left(q_{1}, q_{2}\right) \in \mathbf{R}^{n} \times \mathbf{R}^{n}$ such that

$$
D_{x} q_{1}+D_{z} q_{2}=r .
$$

Since $z$ is a regular value of $\pi \circ \phi=g$ and $\phi$ is differentiable at $\phi^{-1}(\bar{x}, z)$, there exists $w \in \mathbf{R}^{n}$ such that $\left(w, q_{2}\right) \in \operatorname{Im} D \phi\left(\phi^{-1}(\bar{x}, z)\right)$. Let $q=q_{1}-w$. Then $\left(w, q_{2}\right) \in \operatorname{Im} D \phi\left(\phi^{-1}(\bar{x}, z)\right) \Rightarrow D_{x} w+D_{z} q_{2}=0$, so

$$
\begin{aligned}
D_{x} q & =D_{x} q_{1}-D_{x} w+D_{z} q_{2}-D_{z} q_{2}=D_{x} q_{1}+D_{z} q_{2}-\left(D_{x} w+D_{z} q_{2}\right) \\
& =D_{x} q_{1}+D_{z} q_{2}=r .
\end{aligned}
$$

So $y$ is a regular value of $f(\cdot, z)$.

Remark 3.3. The assumptions that $f$ is locally Lipschitz, tame, and Fréchet equidifferentiable in $z$ guarantee that there exists a set $D \subset \mathbf{R}^{n}$ of Lebesgue measure 0 such that

$$
D \times Z \supset\left\{(x, z) \in \mathbf{R}^{n} \times Z: f \text { is not differentiable at }(x, z)\right\} .
$$

Call a function for which there exists such a set $D$ controlled. As will become clear, this result and the remaining results of the paper hold if instead of assuming that $f$ is tame and Fréchet equi-differentiable in $z$, the function $f$ is assumed to be controlled.

To extend this result to the case in which the parameters are drawn from an infinite-dimensional set, Lebesgue measure zero will be replaced with Christensen's ([3] Haar measure zero, equivalently with Hunt, Sauer and Yorke's [9] shyness, as 
the notion of exceptional [4 Anderson and Zame [1] have extended the work of Hunt, Sauer and Yorke and Christensen by defining prevalence and shyness relative to a convex subset which may be a shy subset of the ambient space. Their notion of relative prevalence, presented below, is the concept of generic adopted here.

Definition 3.4. Let $Z$ be a topological vector space and let $C \subset Z$ be a convex Borel subset of $Z$ which is completely metrizable in the relative topology. Let $c \in C$. A universally measurable subset $E \subset Z$ is shy in $C$ at $c$ if for each $\delta>0$ and each neighborhood $W$ of 0 in $Z$, there is a regular Borel probability measure $\mu$ on $Z$ with compact support such that supp $\mu \subset(\delta(C-c)+c) \cap(W+c)$ and $\mu(E+z)=0$ for every $z \in Z$. The set $E$ is shy in $C$ if it is shy at each point $c \in C$. A (not necessarily universally measurable) subset $F \subset C$ is shy in $C$ if it is contained in a shy universally measurable set. A subset $K \subset C$ is prevalent in $C$ if its complement $C \backslash K$ is shy in $C$.

Like Lebesgue measure 0, (relative) shyness is translation invariant, preserved under countable unions, coincides with Lebesgue measure 0 in $\mathbf{R}^{n}$, and no relatively open set is relatively shy. In particular, every relatively prevalent set is dense. One particularly straightforward example of a shy set is a set such that it and all of its translates have Lebesgue measure 0 in some finite-dimensional subspace. Formally:

Definition 3.5. Let $Z$ be a topological vector space and let $C \subset Z$ be a convex Borel subset of $Z$ which is completely metrizable in the relative topology. A universally measurable subset $E \subset C$ is finitely shy in $C$ if there is a finite-dimensional subspace $V \subset Z$ such that $\lambda_{V}(C+a)>0$ for some $a \in Z$ and $\lambda_{V}(E+z)=0$ for every $z \in Z \sqrt[5]{5}$ A (not necessarily universally measurable) subset $F \subset C$ is finitely shy in $C$ if it is contained in a finitely shy universally measurable set. A subset $K \subset C$ is finitely prevalent in $C$ if its complement $C \backslash K$ is finitely shy in $C$.

All finitely prevalent sets in $C$ are also prevalent in $C$ (Anderson and Zame [1, Fact 6]). The finite-dimensional transversality theorem established above then provides the foundation for a finitely prevalent, and thus prevalent, transversality theorem.

As a preliminary step, the following lemma establishes that the set of parameter values $z$ for which $y$ is a regular value of $f(\cdot, z)$ is universally measurable.

Lemma 3.6. Let $Z$ be a Banach space and let $f: \mathbf{R}^{n} \times Z \rightarrow \mathbf{R}^{n}$ be locally Lipschitz, Fréchet equi-differentiable in $z$, and tame. Let $C \subset Z$ be a convex Borel set in $Z$. For a given $y \in \mathbf{R}^{n}, R \equiv\{z \in C: y$ is a regular value of $f(\cdot, z)\}$ is universally measurable.

Proof. Since all analytic sets are universally measurable (Dellacherie and Meyer 7 , III.33(a)]), it suffices to show that $R$ is analytic. Let

$$
S=C \backslash R=\{z \in C: y \text { is not a regular value of } f(\cdot, z)\} \text {. }
$$

\footnotetext{
${ }^{4}$ Another alternative is to use topological notions of genericity such as open and dense or residual. These may be appropriate in some problems, but for problems in which statements are loosely interpreted in a probabilistic sense, concerning the likelihood of a certain event occurring, these topological notions are far from satisfactory. For example, as is well known, even in $\mathbf{R}^{n}$ open and dense sets can have arbitrarily small Lebesgue measure, and residual sets can have Lebesgue measure 0. Stinchcombe [19] explores the relationship between prevalence and probability.

${ }^{5}$ Hunt, Sauer and Yorke 9] also discuss this case, and say that there is a probe for such a set.
} 
Let

$$
B_{1}=\left\{(x, z) \in \mathbf{R}^{n} \times C: f(x, z)=y \text { and } f(\cdot, z) \text { is not differentiable at } x\right\}
$$

and

$$
B_{2}=\left\{(x, z) \in \mathbf{R}^{n} \times C: f(x, z)=y \text { and } D_{x} f(x, z) \text { exists and is singular }\right\} .
$$

Then $S=\pi_{Z}(B)$, where $B=B_{1} \cup B_{2}$ and $\pi_{Z}(\cdot)$ denotes projection onto $Z$. To show that $S$ is analytic, and hence that $R$ is analytic, it suffices to show that $B$ is a Borel set (Dellacherie and Meyer [7, Theorem III.13]).

To that end, first consider the set $B_{1}$ :

$$
\begin{aligned}
B_{1}= & \left\{(x, z) \in \mathbf{R}^{n} \times C: f(x, z)=y\right\} \\
& \cap\left\{(x, z) \in \mathbf{R}^{n} \times C: f(\cdot, z) \text { is not differentiable at } x\right\} \\
= & \left\{(x, z) \in \mathbf{R}^{n} \times C: f(x, z)=y\right\} \\
& \cap\left\{(x, z) \in \mathbf{R}^{n} \times C: f \text { is not differentiable at }(x, z)\right\} \\
= & \left\{(x, z) \in \mathbf{R}^{n} \times C: f(x, z)=y\right\} \cap(D \times C),
\end{aligned}
$$

where $D=\left\{x \in \mathbf{R}^{n}: f\right.$ is not differentiable at $(x, z)$ for some $\left.z\right\}$, and the last two equalities follow because $f$ is Fréchet equi-differentiable in $z$ and tame. Now since $f$ is locally Lipschitz, $\left\{(x, z) \in \mathbf{R}^{n} \times C: f(x, z)=y\right\}$ is a Borel set. By tameness, $D=\left\{x \in \mathbf{R}^{n}: f(\cdot, \hat{z})\right.$ is not differentiable at $\left.x\right\}$ for some fixed $\hat{z} \in Z$, and $f(\cdot, \hat{z}): \mathbf{R}^{n} \rightarrow \mathbf{R}^{n}$ is locally Lipschitz, so $D$ is a Borel set in $\mathbf{R}^{n}$. Thus $D \times C$ is a Borel set, which means that $B_{1}$ is the intersection of two Borel sets and hence Borel. Similarly,

$$
B_{2}=\left\{(x, z) \in \mathbf{R}^{n} \times C: f(x, z)=y\right\} \cap\left\{(x, z) \in D^{c} \times C: D_{x} f(x, z) \text { is singular }\right\} .
$$

To show that $B_{2}$ is Borel it suffices to show that $\left\{(x, z) \in D^{c} \times C: D_{x} f(x, z)\right.$ is singular $\}$ is Borel. But $D_{x} f(\cdot, \cdot)$ is measurable and the set of singular $n \times n$ matrices is closed, so this is a Borel set. Thus $B_{2}$, and hence $B=B_{1} \cup B_{2}$, is Borel.

The following is then the main result of the paper.

Theorem 3.7. Let $Z$ be a Banach space and let $f: \mathbf{R}^{n} \times Z \rightarrow \mathbf{R}^{n}$ be locally Lipschitz, Fréchet equi-differentiable in $z$ and tame. Let $C \subset Z$ be a convex Borel set in $Z$ that is completely metrizable in the relative topology. If $y$ is a strongly regular value of $f$ relative to $C$, then $R \equiv\{z \in C: y$ is a regular value of $f(\cdot, z)\}$ is finitely prevalent in $C$.

Proof. We will show that $S=C \backslash R$ is finitely shy in $C$. By the previous lemma $S$ is universally measurable. Let $V$ be an $n$-dimensional subspace of $Z$ such that $\partial_{w} f^{z}\left(x, z_{V}\right)$ is of maximal rank for each $(x, z) \in f^{-1}(y)$. Such a subspace exists because $y$ is a strongly regular value of $f$. Moreover, by assumption $\exists a \in Z$ such that $\lambda_{V}(C+a)>0$.

Let $z \in Z$ be arbitrary and consider $(S-z) \cap V$. An element $v \in(S-z) \cap V \Longleftrightarrow$ $v \in V$ and $v=\tilde{z}-z$ for some $\tilde{z} \in S$. Then $v \in(S-z) \cap V$ if and only if $y$ is not a regular value of $f(\cdot, v+z)$, or equivalently, if and only if $y$ is not a regular value of $f^{z}\left(\cdot, v+z_{V}\right)$. Thus $v \in(S-z) \cap V$ if and only if $v+z_{V} \in S^{z} \equiv\{w \in$ $V: y$ is not a regular value of $\left.f^{z}(\cdot, w)\right\}$, i.e., if and only if $v \in S^{z}-z_{V}$. But $y$ is a strongly regular value of $f^{z}$, so by Lemma $3.2, S^{z}$ has Lebesgue measure 0 in $V$, and thus $S^{z}-z_{V}$ has Lebesgue measure 0 in $V$. Since $(S-z) \cap V=S^{z}-z_{V}$, 
$(S-z) \cap V$ has Lebesgue measure 0 in $V$. As $z$ was arbitrary, $S$ is finitely shy in $C$.

If $Z=\mathbf{R}^{k}$ and $C \subset \mathbf{R}^{k}$ has nonempty interior, then a set is shy in $C$ if and only if it has Lebesgue measure 0 in $\mathbf{R}^{k}$ (Anderson and Zame ([1, Fact 5]). The following finite-dimensional transversality theorem is then an immediate corollary of the previous results.

Corollary 3.8. Let $f: \mathbf{R}^{n} \times \mathbf{R}^{k} \rightarrow \mathbf{R}^{n}$ be locally Lipschitz, equi-differentiable in $z$ and tame, where $k \geq n$. Let $C \subset \mathbf{R}^{k}$ have nonempty interior. If $y$ is a strongly regular value of $f$, then $R \equiv\{z \in C: y$ is a regular value of $f(\cdot, z)\}$ has full Lebesgue measure.

\section{Applications}

An application in which these questions are of central importance, and to which these results apply, is the study of equilibria in competitive markets. For models with a finite-dimensional commodity space, Debreu's [6] seminal work gives conditions under which the set of equilibria is finite in number and each equilibrium is locally a smooth function of the parameters for all parameter values outside a set of Lebesgue measure 0. Debreu's work relies on Sard's theorem and thus requires the equations characterizing equilibria to be sufficiently smooth. In an infinitedimensional setting, including important classes of dynamic infinite horizon models and continuous-time asset trading models, the equations characterizing equilibria typically fail to be differentiable. Shannon [16, 17] and Shannon and Zame [18] show that the equations characterizing equilibria in these models are instead typically locally Lipschitz continuous under conditions analogous to Debreu's.

These models start with a description of traded commodities and admissible prices via a dual pair $\left\langle X, X^{\prime}\right\rangle$, where $X$ is a partially ordered topological vector space and $X^{\prime}$ is its topological dual 6 For our purposes, suppose in addition that $X$ is a Banach space. In a model with $m+1$ traders, equilibria can be characterized as the vectors $\lambda \in A \subset \mathbf{R}^{m}$ such that $f\left(\lambda, e_{1}, \ldots, e_{m}\right)=0$ for a locally Lipschitz function $f: A \times X^{m} \rightarrow \mathbf{R}^{m}$ taking the form

$$
f\left(\lambda, e_{1}, \ldots, e_{m}\right)=\left(p(\lambda) \cdot\left(x_{1}(\lambda)-e_{1}\right), \ldots, p(\lambda) \cdot\left(x_{m}(\lambda)-e_{m}\right)\right),
$$

where $p: A \rightarrow X_{++}^{\prime}$ is locally bounded (i.e., $p(B)$ is bounded for each bounded set $B \subset A)$ and $x_{i}: A \rightarrow X_{+}$is locally Lipschitz continuous for each $i=1, \ldots, m$. Such a function $f$ is locally Lipschitz continuous, Fréchet equi-differentiable in $\left(e_{1}, \ldots, e_{m}\right)$ and tame. To see how the results of the paper can be applied, let $\bar{e} \in X_{++}$be fixed and set $C \equiv\left\{\left(e_{1}, \ldots, e_{m+1}\right) \in X_{+}^{m+1}: \sum_{i=1}^{m+1} e_{i}=\bar{e}\right\} ; C$ is clearly a convex Borel subset of $X^{m+1}$. The element $e_{i}$ is interpreted as the exogenous initial position of trader $i$. To see that 0 is a strongly regular value of $f$ relative to $C$, choose $y \in X_{+} \backslash\{0\}$ such that $\exists b>0$ for which $\bar{\omega}-b y \in X_{+} \backslash\{0\}$. Let $V \subset X^{m+1}$ be the $m$-dimensional subspace

$$
V \equiv\left\{v \in X^{m+1}: v_{i}=\alpha_{i} y, \text { for some } \alpha_{i} \in \mathbf{R}, i=1, \ldots, m, \text { and } \sum_{i=1}^{m+1} v_{i}=\bar{e}\right\} .
$$

\footnotetext{
${ }^{6}$ Given a dual pair $\left\langle X, X^{\prime}\right\rangle$ where $X$ is partially ordered, $X_{+}=\{x \in X: x \geq 0\}, X_{+}^{\prime}=\left\{x^{\prime} \in\right.$ $X^{\prime}:\left\langle x, x^{\prime}\right\rangle \geq 0$ for all $\left.x \in X_{+}\right\}$, and $X_{++}=\left\{x \in X:\left\langle x, x^{\prime}\right\rangle>0\right.$ for all $\left.x^{\prime} \in X_{+}^{\prime}\right\}$.
} 
For any vector $e=\left(e_{1}, \ldots, e_{m+1}\right) \in X_{++}^{m+1}$ and $\lambda \in A, \partial_{v} f^{e}\left(\lambda, e_{V}\right)=\left\{(p(\lambda) \cdot y) I_{m}\right\}$, where $I_{m}$ is the $m \times m$ identity matrix. Since $p(\lambda)$ is strictly positive for each $\lambda$, $\partial_{v} f^{e}\left(\lambda, e_{V}\right)$ is nonsingular. Also $\lambda_{V}(C)>0$, so 0 is a strongly regular value of $f$ relative to $C$. Let $R \equiv\{e \in C: 0$ is a regular value of $f(\cdot, e)\}$. $R$ is prevalent in $C$ by Theorem 3.7, and by Theorem 2.3, for each $e \in R$ the equilibria of the economy corrsponding to $e$ are determinate in that each equilibrium is locally unique and every selection from the equilibrium set is locally upper Lipschitzian and semidifferentiable at $e$.

\section{REFERENCES}

[1] Anderson, R. and W. Zame (2001), "Genericity with Infinitely Many Parameters," Advances in Theoretical Economics, Vol. 1, No. 1, pp. 1-65. MR2002579(2004g:91038)

[2] Aubin, J.-P. (1984), "Lipschitz Behaviour of Solutions to Convex Minimization Problems," Mathematics of Operations Research, 9, 97-102. MR0736641(86f:90119)

[3] Christensen, J.P.R. (1974), Topology and Borel Structure, North-Holland Mathematical Studies Vol. 10, Amsterdam: North-Holland. MR0348724 (50:1221)

[4] Aarke, F. (1983), Optimization and Nonsmooth Analysis, New York: Wiley Interscience, 1983. MR0709590 (85m:49002)

[5] Clarke, F., Y. Ledyaev, R. Stern and R. Wolenski (1998), Nonsmooth Analysis and Control Theory, New York: Springer-Verlag, 1998. MR1488695 (99a:49001)

[6] Debreu, G. (1970), "Economies with a Finite Set of Equilibria," Econometrica, 38, 387-392. MR0278702 (43:4432)

[7] Dellacherie, C. and P.-A. Meyer (1978), Probabilities and Potential, North-Holland Mathematical Studies Vol. 29, Amsterdam: North-Holland. MR0521810 (80b:60004)

[8] Fort, M. (1950), "Essential and Nonessential Fixed Points," American Journal of Mathematics, 72, 315-320. MR0034573(11:609b)

[9] Hunt, B.R., T. Sauer, and J.A. Yorke (1992), "Prevalence: A Translation-Invariant 'Almost Every' on Infinite-Dimensional Spaces," Bulletin (New Series) of the American Mathematical Society, 27, 217-238. MR.1161274 (93k:28018)

[10] King, A.J. and R.T. Rockafellar (1992), "Sensitivity Analysis for Nonsmooth Generalized Equations," Mathematical Programming, 55, 193-212. MR.1167597(93d:90064)

[11] Rader, T. (1973), "Nice Demand Functions," Econometrica, 41, 913-935. MR0441276 (55:14139)

[12] Rockafellar, R.T. (1988a), "Proto-differentiability of Set-valued Mappings and its Applications in Optimization," Annals of the Institute of H. Poincaré: Analyse Non Lineaire. MR1019126 (90k:90140)

[13] Rockafellar, R.T. (1988b), "First and Second-order Epi-differentiability in Nonlinear Programming," Transactions of the American Mathematical Society, 307, 75-108. MR0936806 (90a:90216)

[14] Rockafellar, R.T. and R. Wets (1999), Variational Analysis, Springer-Verlag. MR 1491362 (98m:49001)

[15] Shannon, C. (1994), "Regular Nonsmooth Equations," Journal of Mathematical Economics, 23(2), 147-165. MR:1266510 (95c:49025)

[16] Shannon, C. (1999), "Determinacy of Competitive Equilibria in Economies with Many Commodities," Economic Theory, 14(1), 29-87. MR1707170(2000j:91045)

[17] Shannon, C. (2002), "Inada Conditions and the Determinacy of Equilibria," preprint, October 2002.

[18] Shannon, C. and W. Zame (2002), "Quadratic Concavity and Determinacy in Economies with Infinitely Many Commodities," Econometrica, 70, 631-662. MR.1913825 (2003f:91071)

[19] Stinchcombe, M. (2001), "The Gap Between Probability and Prevalence: Loneliness in Vector Spaces," Proceedings of the American Mathematical Society, 129, 451-457. MR1694881 (2001e:28022)

Department of Economics and Department of Mathematics, University of CaliforniaBerkeley, Berkeley, California 94720

E-mail address: cshannon@econ.berkeley.edu 